\title{
An FFT Based Fast Poisson Solver on Spherical Shells
}

\author{
Yin-Liang Huang ${ }^{1, *}$, Jian-Guo Liu² and Wei-Cheng Wang ${ }^{3}$ \\ ${ }^{1}$ Department of Applied Mathematics, National University of Tainan, Tainan 70005, \\ Taiwan. \\ ${ }^{2}$ Department of Physics and Department of Mathematics, Duke University, Durham, \\ NC 27708, USA. \\ ${ }^{3}$ Department of Mathematics, National Tsing Hua University, Hsinchu 30013, \\ Taiwan.
}

Received 6 May 2009; Accepted (in revised version) 8 June 2009

Available online 17 September 2010

To the memory of David Gottlieb

\begin{abstract}
We present a fast Poisson solver on spherical shells. With a special change of variable, the radial part of the Laplacian transforms to a constant coefficient differential operator. As a result, the Fast Fourier Transform can be applied to solve the Poisson equation with $\mathcal{O}\left(N^{3} \log N\right)$ operations. Numerical examples have confirmed the accuracy and robustness of the new scheme.
\end{abstract}

AMS subject classifications: 35Q86, 65N06, 65N15, 65N22, 65T50

Key words: Poisson equation, spherical coordinate, FFT, spectral-finite difference method, fast diagonalization, high order accuracy, error estimate, trapezoidal rule, Euler-Maclaurin formula, Bernoulli numbers.

\section{Introduction}

The purpose of this paper is to propose a simple fast solver for the Poisson equation in a spherical shell

$$
\left\{\begin{array}{l}
\frac{\partial_{\rho}\left(\rho^{2} \partial_{\rho} u\right)}{\rho^{2}}+\frac{\partial_{\theta}\left(\sin \theta \partial_{\theta} u\right)}{\rho^{2} \sin \theta}+\frac{\partial_{\phi}^{2} u}{\rho^{2} \sin ^{2} \theta}=f, \text { in } \Omega, \\
\left.u\right|_{\rho=\rho_{\min }}=u^{L}(\theta, \phi), \\
\left.u\right|_{\rho=\rho_{\max }}=u^{R}(\theta, \phi),
\end{array}\right.
$$

*Corresponding author. Email addresses: liang@mail.nutn.edu.tw (Y.-L. Huang), jian-guo.liu@duke.edu (J.-G. Liu), wangwc@math.nthu.edu.tw (W.-C. Wang) 
where

$$
\Omega=\left\{\rho_{\min }<\rho<\rho_{\max }, 0 \leq \theta \leq \pi, 0 \leq \phi \leq 2 \pi\right\} .
$$

The Poisson equation in the spherical shell geometry is important in many geophysical and solar-physical applications $[5,14,15]$.

Eq. (1.1) can be put in a more symmetric form

$$
\left\{\begin{array}{l}
\partial_{\rho}\left(\rho^{2} \partial_{\rho} \sin ^{2} \theta u\right)+\left(\sin \theta \partial_{\theta}\right)^{2} u+\partial_{\phi}^{2} u=\rho^{2}\left(\sin ^{2} \theta\right) f, \text { in } \Omega, \\
\left.u\right|_{\rho=\rho_{\min }}=u^{L}(\theta, \phi), \\
\left.u\right|_{\rho=\rho_{\max }}=u^{R}(\theta, \phi) .
\end{array}\right.
$$

In this symmetric form (1.2), one can apply Fast Fourier Transform to both the $\theta$ and $\phi$ derivatives (see Section 2 for details) to obtain optimal efficiency. The major obstacle for developing an overall fast solver is the radial derivatives which constitute a variable coefficient differential operator. The most popular approaches include Poisson solvers based on FFT in two directions or spherical harmonic functions which requires a Fast Legendre transform $[1,4,6,7,9,12,13,16]$. There are also other approaches using different sets of grids such as the Cubed Sphere grid [11] and the Yin-Yang grid [17].

In this paper, we propose a simple alternative, which provides a more accessible fast solver to (1.2) via FFT in all three variables. We propose the following simultaneous change of dependent and independent variables

$$
\begin{aligned}
& s=\frac{\ln \rho-\ln \rho_{\min }}{\ln \rho_{\max }-\ln \rho_{\min }}, \\
& v=\sqrt{\rho} u .
\end{aligned}
$$

It is easy to see that, under the transformation (1.3), the Poisson equation (1.1) now takes the form

$$
\sin ^{2} \theta\left(\alpha \partial_{s}^{2}-\frac{1}{4}\right) v+\left(\sin \theta \partial_{\theta}\right)^{2} v+\partial_{\phi}^{2} v=g \equiv \rho^{\frac{5}{2}} \sin ^{2} \theta f
$$

where

$$
\alpha=\left(\ln \rho_{\max }-\ln \rho_{\min }\right)^{-2},
$$

with boundary data

$$
\begin{aligned}
& \left.v\right|_{s=0}=v^{L}(\theta, \phi) \equiv \sqrt{\rho_{\min }} u^{L}(\theta, \phi), \\
& \left.v\right|_{s=1}=v^{R}(\theta, \phi) \equiv \sqrt{\rho_{\max }} u^{R}(\theta, \phi) .
\end{aligned}
$$

The significance of the transformation (1.3) is that the radial part now becomes a constant coefficient differential operator. As a consequence, the discretized operator for $\left(\alpha \partial_{s}^{2}-\right.$ $1 / 4)$ can be fast-diagonalized via FFT, resulting in an fast solver with total $\mathcal{O}\left(N^{3} \log N\right)$ 
operation counts. This can be done in the uniform grid setting with second, fourth and higher order methods. We will elaborate on them in the following sections.

To our knowledge, the transformation (1.3) is new. It should be noted, however, the transformation only applies to spherical shells but not the whole sphere as $s$ is not well defined for $\rho_{\min }=0$. In addition, it may be difficult to find similar transformations for the inhomogeneous Helmholtz equation (or modified Poisson equation) $c u-\Delta u=f$.

\section{Fourier expansion in $\theta$ and $\phi$}

A conventional wisdom to avoid confusion caused by the pole singularity is to shift the $\theta$ grids off the pole $(\theta=0, \pi)$ by half a mesh size. The resulting grids is given by

$$
\begin{aligned}
& s_{i}=i \Delta s, \quad 0 \leq i \leq N_{1}, \quad \Delta s=\frac{1}{N_{1}}, \\
& \theta_{j}=\left(j-\frac{1}{2}\right) \Delta \theta, \quad 1 \leq j \leq N_{2}, \quad \Delta \theta=\frac{\pi}{N_{2}}, \\
& \phi_{k}=k \Delta \phi, \quad 1 \leq k \leq N_{3}, \quad \Delta \phi=\frac{2 \pi}{N_{3}} .
\end{aligned}
$$

For each $i, j$, we can expand

$$
v\left(s_{i}, \theta_{j}, \phi_{k}\right)=\sum_{n=1}^{N_{3}} \hat{v}_{n}\left(s_{i}, \theta_{j}\right) \frac{\exp \left(\mathbf{i} n \phi_{k}\right)}{\sqrt{2 \pi}}, \quad g\left(s_{i}, \theta_{j}, \phi_{k}\right)=\sum_{n=1}^{N_{3}} \hat{g}_{n}\left(s_{i}, \theta_{j}\right) \frac{\exp \left(\mathbf{i} n \phi_{k}\right)}{\sqrt{2 \pi}},
$$

with

$$
\begin{aligned}
& \hat{v}_{n}\left(s_{i}, \theta_{j}\right)=\Delta \phi \sum_{k=1}^{N_{3}} v\left(s_{i}, \theta_{j}, \phi_{k}\right) \frac{\exp \left(-\mathbf{i} n \phi_{k}\right)}{\sqrt{2 \pi}}, \\
& \hat{g}_{n}\left(s_{i}, \theta_{j}\right)=\Delta \phi \sum_{k=1}^{N_{3}} g\left(s_{i}, \theta_{j}, \phi_{k}\right) \frac{\exp \left(-\mathbf{i} n \phi_{k}\right)}{\sqrt{2 \pi}} .
\end{aligned}
$$

Substituting (2.2) back to (1.4), we have

$$
\left(\alpha \partial_{s}^{2}-\frac{1}{4}\right) \sin ^{2} \theta \hat{v}_{n}+\left(\sin \theta \partial_{\theta}\right)^{2} \hat{v}_{n}-n^{2} \hat{v}_{n}=\hat{g}_{n}, \quad 1 \leq n \leq N_{3} .
$$

From (2.3) and the identification $v(s,-\theta, \phi)=v(s, \theta, \phi \pm \pi)$, it is easy to derive the following symmetry condition

$$
\hat{v}_{n}(s,-\theta)=(-1)^{n} \hat{v}_{n}(s, \theta), \quad \hat{g}_{n}(s,-\theta)=(-1)^{n} \hat{g}_{n}(s, \theta),
$$

which turns $\hat{v}_{n}(s, \cdot)$ and $\hat{g}_{n}(s, \cdot)$ into $2 N_{2}$-periodic functions. As a result, we can expand them as

$$
\hat{v}_{n}\left(s_{i}, \theta_{j}\right)=\sum_{m=1}^{2 N_{2}} \hat{\hat{v}}_{m, n}\left(s_{i}\right) \frac{\exp \left(\mathbf{i} m \theta_{j}\right)}{\sqrt{2 \pi}}, \quad \hat{g}_{n}\left(s_{i}, \theta_{j}\right)=\sum_{m=1}^{2 N_{2}} \hat{\hat{g}}_{m, n}\left(s_{i}\right) \frac{\exp \left(\mathbf{i} m \theta_{j}\right)}{\sqrt{2 \pi}},
$$


where $\theta_{j}=(j-1 / 2) \Delta \theta,-N_{2}+1 \leq j \leq N_{2}$, and

$$
\begin{aligned}
& \hat{\hat{v}}_{m, n}\left(s_{i}\right)=\Delta \theta \sum_{j=-N_{2}+1}^{N_{2}} \hat{v}_{n}\left(s_{i}, \theta_{j}\right) \frac{\exp \left(-\mathbf{i} m \theta_{j}\right)}{\sqrt{2 \pi}}, \\
& \hat{\hat{g}}_{m, n}\left(s_{i}\right)=\Delta \theta \sum_{j=-N_{2}+1}^{N_{2}} \hat{g}_{n}\left(s_{i}, \theta_{j}\right) \frac{\exp \left(-\mathbf{i} m \theta_{j}\right)}{\sqrt{2 \pi}} .
\end{aligned}
$$

Using the following identities

$$
\begin{gathered}
\sin ^{2}(\theta) \exp (\mathbf{i} m \theta)=-\frac{1}{4}(\exp (\mathbf{i}(m-2) \theta)-2 \exp (\mathbf{i} m \theta)+\exp (\mathbf{i}(m+2) \theta)), \\
\left(\sin \theta \partial_{\theta}\right)^{2} \exp (\mathbf{i} m \theta)=\frac{m}{4}((m-1) \exp (\mathbf{i}(m-2) \theta)-2 m \exp (\mathbf{i} m \theta) \\
+(m+1) \exp (\mathbf{i}(m+2) \theta))
\end{gathered}
$$

one can recast (2.4) as

$$
\left(\alpha \partial_{s}^{2}-\frac{1}{4}\right)(\mathrm{S} \hat{\hat{v}})_{m, n}+(\mathbb{P} \hat{\hat{v}})_{m, n}-n^{2} \hat{\hat{v}}_{m, n}=\hat{\hat{g}}_{m, n},
$$

where $\mathrm{S}$ and $\mathbb{P}$ are $2 \mathrm{~N}_{2} \times 2 \mathrm{~N}_{2}$ matrices acting on the index $m$,

$$
\begin{aligned}
& (\mathrm{S} \hat{\hat{v}})_{m} \equiv \hat{\hat{v}}_{m-2}-2 \hat{\hat{v}}_{m}+\hat{\hat{v}}_{m+2}, \\
& (\mathbb{P} \hat{\hat{v}})_{m} \equiv(m-1)(m-2) \hat{\hat{v}}_{m-2}-2 m^{2} \hat{\hat{v}}_{m}+(m+1)(m+2) \hat{\hat{v}}_{m+2} .
\end{aligned}
$$

Here the subscript $n$ has been omitted in (2.10a) and (2.10b).

Let $\mathbb{D}$ be a discrete approximation of $\partial_{s}^{2}$ acting on the index $\ell$. Suppose that $\mathbb{D}$ can be diagonalized by $\mathbb{Q}$

$$
\mathbb{D}=\mathbb{Q}^{-1} \Lambda \mathbb{Q}
$$

one can rewrite (2.9) as

$$
\left(\left(\left(\alpha \Lambda-\frac{1}{4} \mathbb{I}\right) \otimes S\right) \hat{\hat{v}}\right)_{\ell, m, n}+(\mathbb{P} \hat{\hat{\hat{v}}})_{\ell, m, n}-n^{2} \hat{\hat{\hat{v}}}_{\ell, m, n}=\hat{\hat{\hat{g}}}_{\ell, m, n},
$$

where

$$
\hat{\hat{\hat{v}}}_{\ell, m, n}=(\mathbb{Q} \hat{\hat{v}})_{\ell, m, n}, \quad \hat{\hat{g}}_{\ell, m, n}=(\mathbb{Q} \hat{\hat{g}})_{\ell, m, n} .
$$

In other words, we end up with a tri-diagonal system

$$
\left(\alpha \lambda_{\ell}-\frac{1}{4}\right)(\mathrm{S} \hat{\hat{v}})_{m}+(\mathbb{P} \hat{\hat{v}})_{m}-n^{2} \hat{\hat{v}}_{m}=\hat{\hat{g}}_{m}
$$

for each subscript $(\ell, n)$, which is omitted in (2.13).

With the transformation (1.3), the diagonalization $\mathbb{D}=\mathbb{Q}^{-1} \Lambda \mathbb{Q}$ can be achieved with fast evaluation of the matrix multiplications $Q \hat{\hat{g}}$ and $\mathbb{Q}^{-1} \hat{\hat{v}}$ in several different settings. We will explain them in details in the next section. 


\section{Fast diagonalization of $\partial_{s}^{2}$ using FFT}

To explain the procedure of the diagonalization (2.11) with fast evaluation of matrix multiplication by $\mathbb{Q}$ and $\mathbb{Q}^{-1}$, it suffices to consider the 1D model problem

$$
\left\{\begin{array}{l}
w^{\prime \prime}-\gamma w=z, \quad \text { in }(0,1), \\
w(0)=w^{L}, \quad w(1)=w^{R},
\end{array}\right.
$$

where $\gamma$ is a non-negative constant. We will use this 1D model problem to demonstrate high order finite difference methods with the uniform grids (2.1).

\subsection{2nd order and compact 4th order differencing}

Without loss of generality, we may assume homogeneous data

$$
w^{L}=0=w^{R},
$$

in (3.1). The general inhomogeneous case only requires minor modification.

The second order scheme for (3.1) with homogeneous data $w^{L}=0=w^{R}$ is given by

$$
D_{s}^{2^{\prime}} w_{i}-\gamma w_{i}=z_{i}, \quad 1 \leq i \leq N-1
$$

where the reduced difference operator

$$
D_{s}^{2^{\prime}} w_{i}= \begin{cases}\frac{-2 w_{1}+w_{2}}{(\Delta s)^{2}}, & i=1, \\ D_{s}^{2} w_{i} \equiv \frac{w_{i-1}-2 w_{i}+w_{i+1}}{(\Delta s)^{2}}, & 2 \leq i \leq N-2 \\ \frac{w_{N-2}-2 w_{N-1}}{(\Delta s)^{2}}, & i=N-1,\end{cases}
$$

is adopted to reflect the fact that $w_{0}=0=w_{N}$ are homogeneous boundary data rather than active variables.

Note that

$$
\left\{\left(\sqrt{2} \sin \left(\ell \pi s_{1}\right), \cdots, \sqrt{2} \sin \left(\ell \pi s_{N-1}\right)\right)^{T}, \quad 1 \leq \ell \leq N-1\right\}
$$

constitutes an orthonormal eigen-basis for $D_{s}^{2^{\prime}}$,

$$
\left(D_{s}^{2^{\prime}} \sin (\ell \pi s)\right)_{i}=\lambda_{\ell} \sin \left(\ell \pi s_{i}\right),
$$

with eigenvalues

$$
\lambda_{\ell}=\frac{-4 \sin ^{2}(\ell \pi \Delta s / 2)}{(\Delta s)^{2}} .
$$


We can then diagonalize $D_{s}^{2^{\prime}}$ by expanding $w$ and $z$ with respect to the basis (3.4)

$$
w_{i}=\sqrt{2} \sum_{\ell=1}^{N-1} \hat{w}_{\ell} \sin \left(\ell \pi s_{i}\right), \quad z_{i}=\sqrt{2} \sum_{\ell=1}^{N-1} \hat{z}_{\ell} \sin \left(\ell \pi s_{i}\right), \quad i=1, \cdots, N-1,
$$

where

$$
\hat{w}_{\ell}=\sqrt{2} \Delta s \sum_{i=1}^{N-1} w_{i} \sin \left(\ell \pi s_{i}\right), \quad \hat{z}_{\ell}=\sqrt{2} \Delta s \sum_{i=1}^{N-1} z_{i} \sin \left(\ell \pi s_{i}\right), \quad \ell=1, \cdots, N-1 .
$$

In other words, (3.2) can be diagonalized as

$$
(\Lambda-\gamma \mathbb{I}) \mathbb{Q} w=\mathbb{Q} z
$$

or

$$
\left(\lambda_{\ell}-\gamma\right) \hat{w}_{\ell}=\hat{z}_{\ell}, \quad \ell=1, \cdots, N-1
$$

Here in (3.8a),

$$
\Lambda=\operatorname{diag}\left(\lambda_{1}, \cdots, \lambda_{N-1}\right), \quad \mathbb{Q}_{\ell, i}=\sqrt{2} \sin \left(\ell \pi s_{i}\right), \quad \mathbb{Q}_{i, \ell}^{-1}=\Delta s \sqrt{2} \sin \left(\ell \pi s_{i}\right) .
$$

Multiplication by $\mathbb{Q}$ and $\mathbb{Q}^{-1}$ are given by the discrete sine transform (3.7a) and (3.7b), which can be evaluated via FFT with $\mathcal{O}(N \log N)$ operations.

For inhomogeneous data $w_{0}=w^{L}, w_{N}=w^{R}$, one simply replaces $z_{1}$ by $z_{1}-w^{L} /(\Delta s)^{2}$ and $z_{N-1}$ by $z_{N-1}-w^{R} /(\Delta s)^{2}$ at the right hand side of (3.2), then proceed as the homogeneous case.

The procedure for diagonalizing the compact 4 th order differencing operator is similar. The compact 4 th order approximation of $\partial_{s}^{2}$ is formally given by [2]

$$
\partial_{s}^{2} w=\left(1+\frac{(\Delta s)^{2}}{12} D_{s}^{2}\right)^{-1} D_{s}^{2} w+\mathcal{O}\left((\Delta s)^{4}\right)
$$

Thus, with homogeneous data $w^{L}=0=w^{R}$, the compact 4 th order difference approximation for (3.1) is given by

$$
D_{s}^{2^{\prime}} w_{i}-\gamma\left(1+\frac{(\Delta s)^{2}}{12} D_{s}^{2^{\prime}}\right) w_{i}=\left(1+\frac{(\Delta s)^{2}}{12} D_{s}^{2}\right) z_{i} \equiv \bar{z}_{i}, \quad i=1, \cdots, N-1
$$

Note that the right hand side of (3.11) involves the full difference operator $D_{s}^{2}$. The evaluation of the source term $\bar{z}_{1}$ and $\bar{z}_{N-1}$ requires $z_{0}$ and $z_{N}$, respectively.

Using the same diagonalizing matrices (3.9), one can put (3.11) into diagonal form

$$
\Lambda \mathbb{Q} w-\gamma\left(\mathbb{I}+\frac{(\Delta s)^{2}}{12} \Lambda\right) \mathbb{Q} w=\mathbb{Q} \bar{z}
$$


or componentwise as

$$
\lambda_{\ell} \hat{w}_{\ell}-\gamma\left(1+\frac{(\Delta s)^{2}}{12} \lambda_{\ell}\right) \hat{w}_{\ell}=\hat{z}_{\ell}, \quad 1 \leq \ell \leq N-1
$$

For inhomogeneous data $w_{0}=w^{L}, w_{N}=w^{R}$, one replaces $\bar{z}_{1}$ by $\bar{z}_{1}-w^{L} /(\Delta s)^{2}+\gamma w^{L} / 12$ and $\bar{z}_{N-1}$ by $\bar{z}_{N-1}-w^{R} /(\Delta s)^{2}+\gamma w^{R} / 12$ at the right hand side of (3.11), then proceed as the homogeneous case.

This compact fourth order scheme can be naturally incorporated into the NavierStokes solvers $[3,8]$.

\subsection{Higher order finite difference approximation}

In this section, we continue with an FFT based higher order scheme for the 1D model problem (3.1). To achieve high order accuracy, we split the solution of (3.1) into two parts,

$$
w=\widetilde{W}+W
$$

the homogeneous part

$$
\left\{\begin{array}{l}
W^{\prime \prime}-\gamma W=Z, \quad \text { in }(0,1), \\
W(0)=0, \quad W(1)=0
\end{array}\right.
$$

and the inhomogeneous part

$$
\left\{\begin{array}{l}
\widetilde{W}^{\prime \prime}-\gamma \widetilde{W}=\widetilde{Z}, \quad \text { in }(0,1), \\
\widetilde{W}(0)=w^{L}, \quad \widetilde{W}(1)=w^{R} .
\end{array}\right.
$$

The splitting is performed in such a way that the inhomogeneous solution $\widetilde{W}$ can be constructed easily and explicitly, while the homogeneous part (3.14) can be solved with high accuracy via discrete sine transform. This calls for an additional higher order compatibility condition on the source term $Z$. For a $(2 v)$-th order scheme, this compatibility condition is given by

$$
Z(0)=0=Z(1), \quad Z^{\prime \prime}(0)=0=Z^{\prime \prime}(1), \quad \cdots, \quad Z^{(2 v-4)}(0)=0=Z^{(2 v-4)}(1) .
$$

To be definite, we shall present the 6th order scheme in the rest of this section. Generalization to higher order scheme is straight forward.

\subsubsection{The homogeneous part for the 6th order scheme}

The exact solution to (3.14) can be obtained by expanding both $W$ and $Z$ in sine series. Let

$$
Z(s)=\sqrt{2} \sum_{\ell=1}^{\infty} \widehat{Z}_{\ell} \sin (\ell \pi s),
$$


where

$$
\widehat{Z}_{\ell}=\sqrt{2} \int_{0}^{1} Z(s) \sin (\ell \pi s) d s
$$

denotes the continuous sine transform of $Z$. Thus a solution to (3.14) is given by

$$
W(s)=\sqrt{2} \sum_{\ell=1}^{\infty} \widehat{W}_{\ell} \sin (\ell \pi s) \text {, with } \quad \widehat{W}_{\ell}=\frac{\widehat{Z}_{\ell}}{-\ell^{2} \pi^{2}-\gamma} .
$$

The procedure can be implemented numerically by replacing continuum sine transform with discrete sine transform. That is, we take

$$
W_{i}^{h}=\sqrt{2} \sum_{\ell=1}^{N-1} \hat{W}_{\ell}^{h} \sin \left(\ell \pi s_{i}\right)
$$

where

$$
\begin{aligned}
& \hat{W}_{\ell}^{h}=\frac{\hat{Z}_{\ell}}{-\ell^{2} \pi^{2}-\gamma} \\
& \hat{Z}_{\ell}=\sqrt{2} \Delta s \sum_{i=1}^{N-1} Z\left(s_{i}\right) \sin \left(\ell \pi s_{i}\right) .
\end{aligned}
$$

Note the difference between the hat $\left(^{\wedge}\right)$ and the widehat $\left({ }^{\wedge}\right)$ in (3.17a)-(3.19c). $\widehat{Z}_{\ell}$ and $\widehat{W}_{\ell}, 1 \leq \ell<\infty$, in (3.17a)-(3.18) refer to the continuous Fourier-sine transform of the functions $Z(s)$ and $W(s)$, respectively. While $\hat{Z}_{\ell}$ and $\hat{W}_{\ell}^{h}, 1 \leq \ell \leq N-1$, in (3.19a)-(3.19c) refer to the discrete Fourier-sine transform of the grid functions $\left\{Z\left(s_{i}\right)\right\}_{i=1}^{N-1}$ and $\left\{W_{i}^{h}\right\}_{i=1}^{N-1}$, respectively.

The discrete $L^{2}$-error of the numerical solution is given by

$$
\begin{aligned}
\left\|W-W^{h}\right\|_{L_{h}^{2}(0,1)}^{2} & =\Delta s \sum_{i=1}^{N-1}\left|W_{i}-W_{i}^{h}\right|^{2}=\sum_{\ell=1}^{N-1}\left|\hat{W}_{\ell}-\hat{W}_{\ell}^{h}\right|^{2} \\
& =\sum_{\ell=1}^{N-1}\left(\sqrt{2} \Delta s \sum_{i=1}^{N-1}\left(W\left(s_{i}\right)-\frac{Z\left(s_{i}\right)}{-\ell^{2} \pi^{2}-\gamma}\right) \sin \left(\ell \pi s_{i}\right)\right)^{2},
\end{aligned}
$$

where we have used Plancherel's identity in the second equality of (3.20). Note that

$$
\sqrt{2} \Delta s \sum_{i=1}^{N-1}\left(W\left(s_{i}\right)-\frac{Z\left(s_{i}\right)}{-\ell^{2} \pi^{2}-\gamma}\right) \sin \left(\ell \pi s_{i}\right)
$$

is the trapezoidal approximation of

$$
\sqrt{2} \int_{0}^{1}\left(W(s)-\frac{Z(s)}{-\ell^{2} \pi^{2}-\gamma}\right) \sin (\ell \pi s) d s=\left(\widehat{W}_{\ell}-\frac{\widehat{Z}_{\ell}}{-\ell^{2} \pi^{2}-\gamma}\right)=0 .
$$

Thus, to estimate $\left\|W-W^{h}\right\|_{L_{h}^{2}(0,1)}$, it suffices to estimate the quadrature error of the trapezoidal rule. This is given by the following 
Proposition 3.1. [10] Let $T_{N}$ be the trapezoidal rule approximation of $I=\int_{0}^{1} f(s) \mathrm{d} s$,

$$
T_{N}=\Delta s\left(\frac{1}{2} f(0)+\sum_{i=1}^{N-1} f\left(s_{i}\right)+\frac{1}{2} f(1)\right),
$$

where $\Delta s=1 / N$ and $s_{i}=i \Delta s$. If $f \in C^{2 v}[0,1]$, then

$$
\begin{aligned}
I=T_{N} & -\sum_{\mu=1}^{v} \frac{(\Delta s)^{2 \mu}}{(2 \mu) !} B_{2 \mu}\left(f^{(2 \mu-1)}(1)-f^{(2 \mu-1)}(0)\right) \\
& +(\Delta s)^{2 v} \int_{0}^{1} \widetilde{B}_{2 v}(N s) f^{(2 v)}(s) \mathrm{d} s
\end{aligned}
$$

where the coefficients $B_{2 \mu}$ are the Bernoulli numbers and $\widetilde{B}_{2 v}(x): \mathbb{R} \rightarrow \mathbb{R}$ are given by

$$
\widetilde{B}_{2 v}(x)=2(-1)^{v-1} \sum_{k=1}^{\infty} \frac{\cos (2 \pi k x)}{(2 \pi k)^{2 v}} .
$$

From (3.20), (3.22) and Proposition 3.1,

$$
\begin{aligned}
& \left\|W-W^{h}\right\|_{L_{h}^{2}(0,1)}^{2}=\sum_{\ell=1}^{N-1}\left|\Delta s \sum_{i=1}^{N-1} \xi_{\ell}\left(s_{i}\right)\right|^{2} \\
= & \sum_{\ell=1}^{N-1}\left(\frac{(\Delta s)^{2} B_{2}}{2}\left(\xi_{\ell}^{\prime}(1)-\xi_{\ell}^{\prime}(0)\right)-\frac{(\Delta s)^{4} B_{4}}{24}\left(\xi_{\ell}^{(3)}(1)-\xi_{\ell}^{(3)}(0)\right)+\mathcal{O}\left((\Delta s)^{6}\right)\right)^{2},
\end{aligned}
$$

where

$$
\xi_{\ell}(s)=\sqrt{2}\left(W(s)-\frac{Z(s)}{-\ell^{2} \pi^{2}-\gamma}\right) \sin (\ell \pi s) .
$$

Then the homogeneous Dirichlet condition in (3.14) and the compatibility condition (3.16) with $v=3$ translates to

$$
W(0)=W(1)=W^{\prime \prime}(0)=W^{\prime \prime}(1)=W^{(4)}(0)=W^{(4)}(1)=0 .
$$

Therefore it is easy to see that

$$
\xi_{\ell}^{\prime}(0)=0=\xi_{\ell}^{\prime}(1), \quad \xi_{\ell}^{(3)}(0)=0=\xi_{\ell}^{(3)}(1)
$$

Consequently,

$$
\left\|W-W^{h}\right\|_{L_{h}^{2}(0,1)}=\mathcal{O}\left((\Delta s)^{6}\right)
$$

to leading order. A rigorous error estimate in $L_{h}^{2}$ and $L^{\infty}$ norm can be found in Section 5 . 


\subsubsection{The inhomogeneous part for 6 th order scheme}

The homogeneous boundary condition in (3.14) and the 6th order compatibility condition (3.16) with $v=3$, or equivalently the condition (3.27), gives rise to the following condition for the inhomogeneous solution

$$
\begin{array}{ll}
\widetilde{W}(0)=w(0), & \widetilde{W}(1)=w(1), \\
\widetilde{W}^{\prime \prime}(0)=w^{\prime \prime}(0), & \widetilde{W}^{\prime \prime}(1)=w^{\prime \prime}(1), \\
\widetilde{W}^{(4)}(0)=w^{(4)}(0), & \widetilde{W}^{(4)}(1)=w^{(4)}(1),
\end{array}
$$

which also imply the following identities:

$$
\begin{array}{ll}
\widetilde{Z}(0)=z(0), & \widetilde{Z}(1)=z(1), \\
\widetilde{Z}^{\prime \prime}(0)=z^{\prime \prime}(0), & \widetilde{Z}^{\prime \prime}(1)=z^{\prime \prime}(1) .
\end{array}
$$

It suffices to construct a function $\widetilde{W}(s)$ satisfying (3.29a)-(3.29c) and then generate the source term $\widetilde{Z}$ from (3.15). This can be done easily by taking

$$
\widetilde{W}(s)=a_{0} p_{0}(s)+b_{0} q_{0}(s)+a_{2} p_{2}(s)+b_{2} q_{2}(s)+a_{4} p_{4}(s)+b_{4} q_{4}(s),
$$

where $p_{2 \mu}(s)$ and $q_{2 \mu}(s)$ are elementary functions satisfying

$$
\begin{aligned}
& p_{0}(0)=1, \quad p_{0}(1)=0 \text {; } \\
& q_{0}(0)=0, \quad q_{0}(1)=1 \text {; } \\
& p_{2}(0)=0, \quad p_{2}(1)=0, \quad p_{2}^{\prime \prime}(0)=1, \quad p_{2}^{\prime \prime}(1)=0 \text {; } \\
& q_{2}(0)=0, \quad q_{2}(1)=0, \quad q_{2}^{\prime \prime}(0)=0, \quad q_{2}^{\prime \prime}(1)=1 \text {; } \\
& p_{4}(0)=0, \quad p_{4}(1)=0, \quad p_{4}^{\prime \prime}(0)=0, \quad p_{4}^{\prime \prime}(1)=0, \quad p_{4}^{(4)}(0)=1, \quad p_{4}^{(4)}(1)=0 \text { ； } \\
& q_{4}(0)=0, \quad q_{4}(1)=0, \quad q_{4}^{\prime \prime}(0)=0, \quad q_{4}^{\prime \prime}(1)=0, \quad q_{4}^{(4)}(0)=0, \quad q_{4}^{(4)}(1)=1 .
\end{aligned}
$$

The coefficients $a_{2 \mu}$ and $b_{2 \mu}$ can be systematically calculated as follows.

First, from (3.29a), (3.31) and the first two columns of (3.32a)-(3.32f), we obtain

$$
a_{0}=\widetilde{W}(0)=w(0)=w^{L}, \quad b_{0}=\widetilde{W}(1)=w(1)=w^{R} .
$$

Next, we evaluate $\widetilde{W}^{\prime \prime}(0)$ and $\widetilde{W}^{\prime \prime}(1)$. From (3.31), (3.29b), (3.30a) and the third and fourth columns of (3.32c)-(3.32f), we obtain

$$
\begin{aligned}
& a_{2}=w^{\prime \prime}(0)-a_{0} p_{0}^{\prime \prime}(0)-b_{0} q_{0}^{\prime \prime}(0)=\gamma w^{L}+z(0)-a_{0} p_{0}^{\prime \prime}(0)-b_{0} q_{0}^{\prime \prime}(0), \\
& b_{2}=w^{\prime \prime}(1)-a_{0} p_{0}^{\prime \prime}(1)-b_{0} q_{0}^{\prime \prime}(1)=\gamma w^{R}+z(1)-a_{0} p_{0}^{\prime \prime}(1)-b_{0} q_{0}^{\prime \prime}(1) .
\end{aligned}
$$

In a similar way, we evaluate $\widetilde{W}^{(4)}(0)$ and $\widetilde{W}^{(4)}(1)$ using the last two columns of (3.32e)(3.32f) to get

$$
\begin{aligned}
& \widetilde{W}^{(4)}(0)=a_{0} p_{0}^{(4)}(0)+b_{0} q_{0}^{(4)}(0)+a_{2} p_{2}^{(4)}(0)+b_{2} q_{2}^{(4)}(0)+a_{4} \\
& \widetilde{W}^{(4)}(1)=a_{0} p_{0}^{(4)}(1)+b_{0} q_{0}^{(4)}(1)+a_{2} p_{2}^{(4)}(1)+b_{2} q_{2}^{(4)}(1)+b_{4}
\end{aligned}
$$


In view of (3.29c), we obtain

$$
\begin{aligned}
& a_{4}=w^{(4)}(0)-a_{0} p_{0}^{(4)}(0)-b_{0} q_{0}^{(4)}(0)-a_{2} p_{2}^{(4)}(0)-b_{2} q_{2}^{(4)}(0), \\
& b_{4}=w^{(4)}(1)-a_{0} p_{0}^{(4)}(1)-b_{0} q_{0}^{(4)}(1)-a_{2} p_{2}^{(4)}(1)-b_{2} q_{2}^{(4)}(1) .
\end{aligned}
$$

Here, $w^{(4)}(0)$ and $w^{(4)}(1)$ can be expressed in terms of the boundary data and the source term by taking $\partial_{s}^{2}$ on both sides of (3.1)

$$
\begin{aligned}
& w^{(4)}(0)=\gamma w^{\prime \prime}(0)+z^{\prime \prime}(0)=\gamma^{2} w^{L}+\gamma z(0)+z^{\prime \prime}(0), \\
& w^{(4)}(1)=\gamma w^{\prime \prime}(1)+z^{\prime \prime}(1)=\gamma^{2} w^{R}+\gamma z(1)+z^{\prime \prime}(1) .
\end{aligned}
$$

In practice, one can simplify the expressions $a_{2 \mu}, b_{2 \mu}$ by further imposing

$$
p_{2 \mu}^{(2 \mu+2)}(s)=0, \quad q_{2 \mu}^{(2 \mu+2)}(s)=0, \quad \text { for } \mu=0,1,2,
$$

which, together with (3.32a)-(3.32f), determines the functions $p_{2 \mu}$ and $q_{2 \mu}$ uniquely. The answer is given by

$$
\begin{array}{ll}
p_{0}(s)=1-s, & q_{0}(s)=s, \\
p_{2}(s)=\frac{1}{6}\left((1-s)^{3}-(1-s)\right), & q_{2}(s)=\frac{1}{6}\left(s^{3}-s\right), \\
p_{4}(s)=\frac{(1-s)^{5}}{120}-\frac{(1-s)^{3}}{36}+\frac{7(1-s)}{360}, & q_{4}(s)=\frac{s^{5}}{120}-\frac{s^{3}}{36}+\frac{7 s}{360} .
\end{array}
$$

With (3.38), the coefficients in (3.34) and (3.36) can be further simplified as

$$
\begin{aligned}
& a_{2}=w^{\prime \prime}(0)=\gamma w^{L}+z(0), \quad b_{2}=w^{\prime \prime}(1)=\gamma w^{R}+z(1), \\
& a_{4}=w^{(4)}(0)=\gamma^{2} w^{L}+\gamma z(0)+z^{\prime \prime}(0), \quad b_{4}=w^{(4)}(1)=\gamma^{2} w^{R}+\gamma z(1)+z^{\prime \prime}(1) .
\end{aligned}
$$

The right hand side of (3.15) can now be explicitly calculated

$$
\widetilde{Z}(s)=\widetilde{W}^{\prime \prime}(s)-\gamma \widetilde{W}(s), \quad \text { for } s \in[0,1] .
$$

The right hand side of the homogeneous part (3.14) is therefore given by

$$
Z(s)=z(s)-\widetilde{Z}(s), \quad s \in[0,1] .
$$

The above procedure can be applied to the full equation

$$
\left(\alpha \partial_{s}^{2}-\frac{1}{4}\right) \sin ^{2} \theta \hat{v}_{n}(s, \theta)+\left(\sin \theta \partial_{\theta}\right)^{2} \hat{v}_{n}(s, \theta)-n^{2} \hat{v}_{n}(s, \theta)=\hat{g}_{n}(s, \theta), \quad 1 \leq n \leq N_{3} .
$$

For each $n$, we first split $\hat{v}_{n}$ and $\hat{g}_{n}$ into inhomogeneous and homogeneous parts by

$$
\hat{v}_{n}=\widetilde{\hat{V}}_{n}+\hat{V}_{n} \quad \text { and } \quad \hat{g}_{n}=\widetilde{\hat{G}}_{n}+\hat{G}_{n},
$$


with

$$
\widetilde{\hat{G}}_{n}(0, \theta)=\hat{g}_{n}(0, \theta) \text { and } \widetilde{\hat{G}}_{n}(1, \theta)=\hat{g}_{n}(1, \theta) .
$$

The equation for inhomogeneous part is given by

$$
\left\{\begin{array}{l}
\left(\alpha \partial_{s}^{2}-\frac{1}{4}\right) \sin ^{2} \theta \widetilde{\hat{V}}_{n}+\left(\sin \theta \partial_{\theta}\right)^{2} \widetilde{\hat{V}}_{n}-n^{2} \widetilde{\hat{V}}_{n}=\widetilde{\hat{G}}_{n}, \\
\widetilde{\hat{V}}_{n}(0, \theta)=\hat{v}_{n}^{L}(\theta), \quad \widetilde{\hat{V}}_{n}(1, \theta)=\hat{v}_{n}^{R}(\theta) .
\end{array}\right.
$$

As in (3.31), the inhomogeneous part of (3.43) for the 6th-order scheme is given by

$$
\widetilde{\hat{V}}_{n}(s, \theta)=\sum_{\mu=0}^{2}\left(a_{2 \mu}(\theta) p_{2 \mu}(s)+b_{2 \mu}(\theta) q_{2 \mu}(s)\right) \text {. }
$$

Note that the coefficients $a_{2 \mu}$ and $b_{2 \mu}$ in (3.46) are now functions of $\theta$. With $p_{2 \mu}(s)$ and $q_{2 \mu}(s)$ given by (3.39), the coefficients can be solved explicitly as

$$
\left\{\begin{array}{l}
a_{0}(\theta)=\hat{v}_{n}^{L}(\theta), \\
b_{0}(\theta)=\hat{v}_{n}^{R}(\theta), \\
a_{2}(\theta)=\partial_{s}^{2} \hat{v}_{n}(0, \theta)=\left(\alpha \sin ^{2} \theta\right)^{-1}\left(\hat{g}_{n}(0, \theta)+\left(n^{2}+\frac{\sin ^{2} \theta}{4}\right) a_{0}(\theta)-\left(\sin \theta \partial_{\theta}\right)^{2} a_{0}(\theta)\right), \\
b_{2}(\theta)=\partial_{s}^{2} \hat{v}_{n}(1, \theta)=\left(\alpha \sin ^{2} \theta\right)^{-1}\left(\hat{g}_{n}(1, \theta)+\left(n^{2}+\frac{\sin ^{2} \theta}{4}\right) b_{0}(\theta)-\left(\sin \theta \partial_{\theta}\right)^{2} b_{0}(\theta)\right), \\
a_{4}(\theta)=\partial_{s}^{(4)} \hat{v}_{n}(0, \theta)=\left(\alpha \sin ^{2} \theta\right)^{-1}\left(\partial_{s}^{2} \hat{g}_{n}(0, \theta)+\left(n^{2}+\frac{\sin ^{2} \theta}{4}\right) a_{2}(\theta)-\left(\sin \theta \partial_{\theta}\right)^{2} a_{2}(\theta)\right), \\
b_{4}(\theta)=\partial_{s}^{(4)} \hat{v}_{n}(1, \theta)=\left(\alpha \sin ^{2} \theta\right)^{-1}\left(\partial_{s}^{2} \hat{g}_{n}(1, \theta)+\left(n^{2}+\frac{\sin ^{2} \theta}{4}\right) b_{2}(\theta)-\left(\sin \theta \partial_{\theta}\right)^{2} b_{2}(\theta)\right) .
\end{array}\right.
$$

The equation for the homogeneous part is

$$
\left\{\begin{array}{l}
\left(\alpha \partial_{s}^{2}-\frac{1}{4}\right) \sin ^{2} \theta \hat{V}_{n}+\left(\sin \theta \partial_{\theta}\right)^{2} \hat{V}_{n}-n^{2} \hat{V}_{n}=\hat{G}_{n} \\
\hat{V}_{n}(0, \theta)=0, \quad \hat{V}_{n}(1, \theta)=0
\end{array}\right.
$$

with

$$
\hat{G}_{n}=\hat{g}_{n}-\widetilde{\hat{G}}_{n}=\hat{g}_{n}-\left(\left(\alpha \partial_{s}^{2}-\frac{1}{4}\right) \sin ^{2} \theta \widetilde{\hat{V}}_{n}+\left(\sin \theta \partial_{\theta}\right)^{2} \widetilde{\hat{V}}_{n}-n^{2} \widetilde{\hat{V}}_{n}\right)
$$

Following the same derivation as (2.4)-(2.12), one can transform (3.47a) to the following system:

$$
\left(\left(\left(\alpha \Lambda-\frac{1}{4} \mathbb{I}\right) \otimes S\right) \hat{\hat{V}}\right)_{\ell, m, n}+(\mathbb{P} \hat{\hat{V}})_{\ell, m, n}-n^{2} \hat{\hat{V}}_{\ell, m, n}=\hat{\hat{G}}_{\ell, m, n}
$$


which is a tri-diagonal system

$$
\left(-\alpha \ell^{2} \pi^{2}-\frac{1}{4}\right)(\mathrm{S} \hat{\hat{V}})_{m}+(\mathbb{P} \hat{\hat{V}})_{m}-n^{2} \hat{\hat{V}}_{m}=\hat{\hat{G}}_{m}
$$

for each fixed $(\ell, n)$.

We summarize the 6th-order scheme for (1.4) as the following algorithm.

Algorithm 3.1: The 6th-order solver for $\left(\alpha \partial_{s}^{2}-1 / 4\right)\left(\sin ^{2} \theta v\right)+\left(\sin \theta \partial_{\theta}\right)^{2} v+\partial_{\phi}^{2} v=g$

1. Compute $\hat{g}_{n}\left(s_{i}, \theta_{j}\right), \hat{v}_{n}^{L}\left(\theta_{j}\right), \hat{v}_{n}^{R}\left(\theta_{j}\right)$ by forward FFT in $\phi$ using (2.3),

2. Extend $\hat{g}_{n}\left(s_{i}, \theta_{j}\right), \hat{v}_{n}^{L}\left(\theta_{j}\right), \hat{v}_{n}^{R}\left(\theta_{j}\right)$ using (2.5),

3. Solve $\left(\alpha \partial_{s}^{2}-1 / 4\right)\left(\sin ^{2} \theta \hat{v}_{n}\right)+\left(\sin \theta \partial_{\theta}\right)^{2} \hat{v}_{n}-n^{2} \hat{v}_{n}=\hat{g}_{n}$ :

for $n=1, \cdots, N_{3}$ do

(i) Compute the inhomogeneous part $\widetilde{\hat{V}}_{n}$ from $\hat{v}_{n}^{L}(\theta), \hat{v}_{n}^{R}(\theta)$ and $\hat{g}_{n}(s, \theta)$ using (3.46),

(ii) Compute $\hat{G}_{n}\left(s_{i}, \theta_{j}\right)$, the source term for the homogeneous part, using $(3.47 \mathrm{~b})$,

(iii) Solve the homogeneous part $\hat{V}_{n}\left(s_{i}, \theta_{j}\right)$ using (3.47a):

(a) Compute $\hat{\hat{G}}_{m, n}\left(s_{i}\right)$ by forward FFT in $\theta$ using (2.7),

(b) Compute $\hat{\hat{G}}_{\ell, m, n}$ by forward FST (Fast Sine-Transform) in $s$ using (3.19c):

for $\ell=1, \cdots, N_{1}$ do

Solve $\left\{\hat{\hat{V}}_{\ell, m, n}\right\}_{m=1}^{2 N_{2}}$ from (3.49) with a tri-diagonal solver,

end for

(c) Compute $\hat{\hat{V}}_{m, n}\left(s_{i}\right)$ from $\left\{\hat{\hat{V}}_{\ell, m, n}\right\}_{\ell=1}^{N_{1}}$ by backward FST in $s$ using (3.19a),

(d) Compute $\hat{V}_{n}\left(s_{i}, \theta_{j}\right)$ from $\left\{\hat{V}_{m, n}\left(s_{i}\right)\right\}_{m=1}^{2 N_{2}}$ by backward FFT in $\theta$ using (2.6),

end for

4. Compute $v\left(s_{i}, \theta_{j}, \phi_{k}\right)$ from $\left\{\widetilde{\hat{V}}_{n}\left(s_{i}, \theta_{j}\right)+\hat{V}_{n}\left(s_{i}, \theta_{j}\right)\right\}_{n=1}^{N_{3}}$ by backward FFT in $\phi$ using (2.2).

\section{Numerical results}

In this section, we report standard accuracy check for the 6th order scheme. In both examples below, we take $\Omega=\{2 \leq \rho \leq 4\}$.

\section{Example 4.1.}

$$
\begin{aligned}
& u^{e}(x, y, z)=x y^{2} \sin z+\exp \left(\frac{x^{2}}{16}\right)+x^{3} \\
& f(x, y, z)=\nabla^{2} u^{e}(x, y, z)=\left(2 x-x y^{2}\right) \sin z+\left(\frac{1}{8}+\frac{x^{2}}{64}\right) \exp \left(\frac{x^{2}}{16}\right)+6 x
\end{aligned}
$$




\section{Example 4.2.}

$$
\begin{aligned}
& u^{e}(x, y, z)=x^{2} y^{3} \cos z+\exp (x z), \\
& f(x, y, z)=\nabla^{2} u^{e}(x, y, z)=\left(2 y^{3}+6 x^{2} y-x^{2} y^{3}\right) \cos z+\left(x^{2}+z^{2}\right) \exp (x z) .
\end{aligned}
$$

The relative errors and orders of convergence are reported in Tables 1 and 2. Note that the discretization in $\theta$ and $\phi$ variables have spectral accuracy, therefore $N_{2}$ and $N_{3}$ are held fixed in Tables 1 and 2.

Table 1: Relative error and rate of convergence for Example 4.1.

\begin{tabular}{||c|c|c|c|c||}
\hline$N_{1} \times N_{2} \times N_{3}$ & $\left\|u^{e}-u^{h}\right\|_{\infty} /\left\|u^{\mathcal{e}}\right\|_{\infty}$ & Order & $\left\|u^{e}-u^{h}\right\|_{L^{2}} /\left\|u^{e}\right\|_{L^{2}}$ & Order \\
\hline $32 \times 32 \times 64$ & $2.33 E-9$ & & $1.58 E-9$ & \\
\hline $64 \times 32 \times 64$ & $3.96 E-11$ & 5.83 & $2.63 E-11$ & 5.88 \\
\hline $128 \times 32 \times 64$ & $6.45 E-13$ & 5.92 & $4.23 E-13$ & 5.94 \\
\hline
\end{tabular}

Table 2: Relative error and rate of convergence for Example 4.2.

\begin{tabular}{||c|c|c|c|c||}
\hline$N_{1} \times N_{2} \times N_{3}$ & $\left\|u^{e}-u^{h}\right\|_{\infty} /\left\|u^{e}\right\|_{\infty}$ & Order & $\left\|u^{e}-u^{h}\right\|_{L^{2}} /\left\|u^{e}\right\|_{L^{2}}$ & Order \\
\hline $64 \times 64 \times 64$ & $3.86 E-7$ & & $4.40 E-7$ & \\
\hline $128 \times 64 \times 64$ & $6.66 E-9$ & 5.73 & $7.68 E-9$ & 5.78 \\
\hline $256 \times 64 \times 64$ & $1.10 E-10$ & 5.86 & $1.27 E-10$ & 5.89 \\
\hline
\end{tabular}

\section{Error estimate for the 6th order scheme}

In this section, we give an error estimate of our scheme (3.19) for the homogeneous problem (3.14) with 6th order compatibility condition (3.16) with $v=3$. The argument generalizes easily to higher order cases.

Theorem 5.1. Let $\left\{W_{i}^{h}\right\}_{i=1}^{N-1}$ be the numerical solution of the homogeneous equation (3.14) given by (3.19), with $Z \in H^{6}[0,1]$ satisfying

$$
Z(0)=Z(1)=Z^{\prime \prime}(0)=Z^{\prime \prime}(1)=0 .
$$

Then there exits a constant $C$ independent of $N$ and $Z$, such that

$$
\begin{aligned}
& \sup _{1 \leq i \leq N-1}\left|W\left(s_{i}\right)-W_{i}^{h}\right| \leq C\left(\|Z\|_{C^{4}[0,1]} \ln N+\|Z\|_{H^{6}(0,1)}\right) N^{-6}, \\
& \left\|W\left(s_{i}\right)-W_{i}^{h}\right\|_{L_{h}^{2}(0,1)} \leq C\|Z\|_{H^{6}(0,1)} N^{-6} .
\end{aligned}
$$

Proof. We start with the proof of (5.2a). Without loss of generality, we may assume $Z \in$ $C^{6}[0,1]$. The $H^{6}(0,1)$ case follows from standard density argument. From (3.18), (3.19a) 
and (3.19b), we have

$$
\begin{aligned}
\left|W\left(s_{i}\right)-W_{i}^{h}\right| & =\left|\sqrt{2} \sum_{\ell=1}^{\infty} \widehat{W}_{\ell} \sin \left(\ell \pi s_{i}\right)-\sqrt{2} \sum_{\ell=1}^{N-1} \hat{W}_{\ell}^{h} \sin \left(\ell \pi s_{i}\right)\right| \\
& \leq \sqrt{2} \sum_{\ell=1}^{N-1}\left|\widehat{W}_{\ell}-\hat{W}_{\ell}^{h}\right|+\sqrt{2} \sum_{\ell=N}^{\infty}\left|\widehat{W}_{\ell}\right| \\
& =\sqrt{2} \sum_{\ell=1}^{N-1}\left|\frac{\widehat{Z}_{\ell}-\hat{Z}_{\ell}}{\ell^{2} \pi^{2}+\gamma}\right|+\sqrt{2} \sum_{\ell=N}^{\infty}\left|\frac{\widehat{Z}_{\ell}}{\ell^{2} \pi^{2}+\gamma}\right| \equiv(\mathbf{I})+(\mathbf{I I}) .
\end{aligned}
$$

From $(3.17 b)$ and $(3.19 \mathrm{c})$, we see that $\hat{Z}_{\ell}$ is the trapezoidal approximation of $\widehat{Z}_{\ell}$. In view of Proposition 3.1, we take

$$
f(s)=\zeta_{\ell}(s) \triangleq Z(s) \sqrt{2} \sin (\ell \pi s) \in C^{6}[0,1],
$$

and $v=3$ to get

$$
\frac{\left|\widehat{Z}_{\ell}-\hat{Z}_{\ell}\right|}{\ell^{2} \pi^{2}+\gamma} \leq N^{-6} \frac{B_{6}}{6 !} \frac{\left|\zeta_{\ell}^{(5)}(1)-\zeta_{\ell}^{(5)}(0)\right|}{\left(\ell^{2} \pi^{2}+\gamma\right)}+N^{-6}\left|\int_{0}^{1} \sum_{k=1}^{\infty} \frac{2 \cos (2 \pi k N s)}{(2 \pi k)^{6}\left(\ell^{2} \pi^{2}+\gamma\right)} \zeta_{\ell}^{(6)}(s) \mathrm{d} s\right|,
$$

where we have used (3.24) and the fact that

$$
\zeta_{\ell}^{\prime}(0)=\zeta_{\ell}^{\prime}(1)=\zeta_{\ell}^{(3)}(0)=\zeta_{\ell}^{(3)}(1)=0
$$

Since

$$
\left|\zeta_{\ell}^{(5)}(1)-\zeta_{\ell}^{(5)}(0)\right|=\left|(-1)^{\ell} 5 \sqrt{2} \ell \pi Z^{(4)}(1)-5 \sqrt{2} \ell \pi Z^{(4)}(0)\right| \leq 10 \sqrt{2} \ell\|Z\|_{C^{4}[0,1]},
$$

the first term on the right hand side of (5.4) can be estimated by

$$
\begin{aligned}
\sum_{\ell=1}^{N-1} \frac{N^{-6} B_{6}}{6 !} \frac{\left|\zeta_{\ell}^{(5)}(1)-\zeta_{\ell}^{(5)}(0)\right|}{\left(\ell^{2} \pi^{2}+\gamma\right)} & \leq C\|Z\|_{C^{4}[0,1]} N^{-6} \sum_{\ell=1}^{N-1}\left|\frac{\ell}{\ell^{2} \pi^{2}+\gamma}\right| \\
& \leq C\|Z\|_{C^{4}[0,1]} N^{-6} \ln N .
\end{aligned}
$$

We now continue on the second term on the right hand side of (5.4). We first expand

$$
\zeta_{\ell}^{(6)}(s)=\sqrt{2} \sum_{\mu=0}^{3} c_{\mu} \ell^{6-2 \mu} Z^{(2 \mu)}(s) \sin (\ell \pi s)+\sqrt{2} \sum_{\mu=0}^{2} d_{\mu} \ell^{5-2 \mu} Z^{(2 \mu+1)}(s) \cos (\ell \pi s),
$$

where $c_{\mu}, d_{\mu} \in \mathbb{R}$. Then use the identities

$$
\begin{aligned}
& 2 \cos (2 \pi k N s) \sin (\ell \pi s)=\sin ((2 k N+\ell) \pi s)-\sin ((2 k N-\ell) \pi s), \\
& 2 \cos (2 \pi k N s) \cos (\ell \pi s)=\cos ((2 k N+\ell) \pi s)+\cos ((2 k N-\ell) \pi s),
\end{aligned}
$$


to obtain

$$
\begin{aligned}
& \sqrt{2} \int_{0}^{1} 2 \cos (2 \pi k N s) Z^{(2 \mu)}(s) \sin (\ell \pi s) d s=\left(\widehat{Z^{(2 \mu)}} 2 k N+\ell-\widehat{Z^{(2 \mu)}} 2 k N-\ell\right), \\
& \sqrt{2} \int_{0}^{1} 2 \cos (2 \pi k N s) Z^{(2 \mu+1)}(s) \cos (\ell \pi s) d s=\left(\widehat{Z^{(2 \mu+1)}} 2 k N+\ell+\widehat{Z^{(2 \mu+1)}} 2 k N-\ell\right),
\end{aligned}
$$

where

$$
\widehat{Z^{(v)}} \ell \equiv \begin{cases}\sqrt{2} \int_{0}^{1} Z^{(v)}(s) \sin (\ell \pi s) d s, & \text { if } v \text { is even, } \\ \sqrt{2} \int_{0}^{1} Z^{(v)}(s) \cos (\ell \pi s) d s, & \text { if } v \text { is odd. }\end{cases}
$$

In view of (5.7), it suffices to estimate for $0 \leq \mu \leq 3$,

$$
\begin{aligned}
& \sum_{\ell=1}^{N-1}\left|\sqrt{2} \int_{0}^{1} \sum_{k=1}^{\infty} \frac{2 \cos (2 \pi k N s)}{k^{6}\left(\ell^{2} \pi^{2}+\gamma\right)} \ell^{6-2 \mu} Z^{(2 \mu)}(s) \sin (\ell \pi s) d s\right| \\
= & \sum_{\ell=1}^{N-1}\left|\sqrt{2} \sum_{k=1}^{\infty} \frac{\ell^{6-2 \mu}}{k^{6}\left(\ell^{2} \pi^{2}+\gamma\right)} \int_{0}^{1} 2 \cos (2 \pi k N s) Z^{(2 \mu)}(s) \sin (\ell \pi s) d s\right| \\
= & \sum_{\ell=1}^{N-1}\left|\sum_{k=1}^{\infty} \frac{\ell^{6-2 \mu}}{k^{6}\left(\ell^{2} \pi^{2}+\gamma\right)}\left(\widehat{Z^{(2 \mu)}}{ }_{2 k N+\ell}-\widehat{Z^{(2 \mu)}} 2 k N-\ell\right)\right| \\
\leq & \sum_{\ell=-N+1}^{N-1} \sum_{k=1}^{\infty} \frac{\ell^{6-2 \mu}}{k^{6}\left(\ell^{2} \pi^{2}+\gamma\right)}\left|\widehat{Z^{(2 \mu)}} 2 k N+\ell\right|,
\end{aligned}
$$

and for $\mu=0,1,2$,

$$
\begin{aligned}
& \sum_{\ell=1}^{N-1}\left|\sqrt{2} \int_{0}^{1} \sum_{k=1}^{\infty} \frac{2 \cos (2 \pi k N s)}{k^{6}\left(\ell^{2} \pi^{2}+\gamma\right)} \ell^{5-2 \mu} Z^{(2 \mu+1)}(s) \cos (\ell \pi s) d s\right| \\
\leq & \sum_{\ell=-N+1}^{N-1} \sum_{k=1}^{\infty} \frac{|\ell|^{5-2 \mu}}{k^{6}\left(\ell^{2} \pi^{2}+\gamma\right)}\left|\widehat{Z^{(2 \mu+1)}} 2 k N+\ell\right| .
\end{aligned}
$$

To proceed with the above estimates, we note from (5.10) and integration by parts that

$$
\begin{aligned}
& {\widehat{Z^{(0)}}}_{\ell}=\frac{1}{\ell \pi} \widehat{Z^{(1)}} \ell=\frac{-1}{(\ell \pi)^{2}}{\widehat{Z^{(2)}}}_{\ell}=\frac{-1}{(\ell \pi)^{3}} \widehat{Z}^{(3)} \ell=\frac{1}{(\ell \pi)^{4}}{\widehat{Z^{(4)}}}_{\ell}, \\
& \widehat{Z^{(4)}} \ell=\frac{1}{\ell \pi}\left(\widehat{Z^{(5)}} \ell-\sqrt{2}\left((-1)^{\ell} Z^{(4)}(1)-Z^{(4)}(0)\right)\right), \\
& \widehat{Z^{(5)}} \ell=\frac{-1}{\ell \pi} \widehat{Z^{(6)}} \ell .
\end{aligned}
$$

Consequently,

$$
\begin{aligned}
& \left|\widehat{Z^{(v)}}\right| \leq \frac{1}{(\ell \pi)^{6-v}}\left|\widehat{Z^{(6)}} \ell\right|+\frac{C}{(\ell \pi)^{5-v}}\|Z\|_{C^{4}[0,1]}, \quad 0 \leq v \leq 4, \\
& \left|\widehat{Z^{(5)}}\right|=\frac{1}{\ell \pi} \mid \widehat{Z^{(6)}} \ell .
\end{aligned}
$$


We now substitute (5.14) back to (5.11) and (5.12). Since $|\ell|<2 k N+\ell$, we have for $0 \leq v \leq 4$,

$$
\begin{aligned}
& \sum_{\ell=-N+1}^{N-1} \sum_{k=1}^{\infty} \frac{|\ell|^{6-v}}{k^{6}\left(\ell^{2} \pi^{2}+\gamma\right)}\left|\widehat{Z^{(v)}} 2 k N+\ell\right| \\
\leq & \sum_{\ell=-N+1}^{N-1} \sum_{k=1}^{\infty} \frac{|\ell|^{6-v}}{k^{6}\left(\ell^{2} \pi^{2}+\gamma\right)}\left(\frac{1}{((2 k N+\ell) \pi)^{6-v}}\left|\widehat{Z^{(6)}} 2 k N+\ell\right|+\frac{C}{((2 k N+\ell) \pi)^{5-v}}\|Z\|_{C^{4}[0,1]}\right) \\
\leq & \sum_{\ell=-N+1}^{N-1} \sum_{k=1}^{\infty} \frac{1}{k^{6}((2 k N+\ell) \pi)^{2}}\left|\widehat{Z^{(6)}} 2 k N+\ell\right|+\sum_{\ell=-N+1}^{N-1} \sum_{k=1}^{\infty} \frac{C}{k^{6}((2 k N+\ell) \pi)}\|Z\|_{C^{4}[0,1]} \\
\leq & C\left(\sum_{\ell=-N+1}^{N-1} \sum_{k=1}^{\infty}\left|\frac{1}{((2 k N+\ell) \pi)^{2}}\right|^{2}\right)^{\frac{1}{2}}\left(\sum_{\ell=-N+1}^{N-1} \sum_{k=1}^{\infty}\left|\widehat{Z^{(6)}} 2 k N+\ell\right|^{2}\right)^{\frac{1}{2}}+C \sum_{\ell=-N+1}^{N-1} \sum_{k=1}^{\infty} \frac{\|Z\|_{C^{4}[0,1]}}{k^{6} N \pi} \\
\leq & C\left(\|Z\|_{H^{6}(0,1)}+\|Z\|_{C^{4}[0,1]}\right) \leq C\|Z\|_{H^{6}(0,1)} .
\end{aligned}
$$

Similarly, for $v=5,6$, we also have

$$
\sum_{\ell=-N+1}^{N-1} \sum_{k=1}^{\infty} \frac{|\ell|^{6-v}}{k^{6}\left(\ell^{2} \pi^{2}+\gamma\right)}\left|\widehat{Z^{(v)}} 2 k N+\ell\right| \leq C\|Z\|_{H^{6}(0,1)} .
$$

Therefore

$$
\sum_{\ell=1}^{N-1}\left|\int_{0}^{1} \sum_{k=1}^{\infty} \frac{2 \cos (2 \pi k N s)}{(2 \pi k)^{6}\left(\ell^{2} \pi^{2}+\gamma\right)} \zeta_{\ell}^{(6)}(s) \mathrm{d} s\right| \leq C\|Z\|_{H^{6}(0,1)} .
$$

From (5.4), (5.6) and (5.17), we obtain

$$
(\mathbf{I}) \leq C N^{-6}\left(\|Z\|_{C^{4}[0,1]} \ln N+\|Z\|_{H^{6}(0,1)}\right) .
$$

On the other hand,

$$
\begin{aligned}
(\mathbf{I I})=\sqrt{2} \sum_{\ell=N}^{\infty}\left|\frac{\widehat{Z}_{\ell}}{\ell^{2} \pi^{2}+\gamma}\right| & \leq \sqrt{2}\left(\sum_{\ell=N}^{\infty}\left|\frac{1}{\left(1+\ell^{2}\right)^{3}\left(\ell^{2} \pi^{2}+\gamma\right)}\right|^{2}\right)^{\frac{1}{2}}\left(\sum_{\ell=N}^{\infty}\left(1+\ell^{2}\right)^{3}\left|\widehat{Z}_{\ell}\right|^{2}\right)^{\frac{1}{2}} \\
& \leq C\|Z\|_{H^{3}(0,1)} N^{-7}
\end{aligned}
$$

In view of (5.3), (5.18) and (5.19), the proof for (5.2a) is complete.

The proof for (5.2b) is similar. From (3.19b), (3.19c) we have

$$
\left\|W\left(s_{i}\right)-W_{i}^{h}\right\|_{L_{h}^{2}(0,1)}^{2}=\sum_{\ell=1}^{N-1}\left|\hat{W}_{\ell}-\hat{W}_{\ell}^{h}\right|^{2}=\sum_{\ell=1}^{N-1}\left|\Delta s \sum_{i=1}^{N-1} \xi_{\ell}\left(s_{i}\right)\right|^{2}
$$

where

$$
\xi_{\ell}(s)=\left(W(s)-\frac{Z(s)}{-\ell^{2} \pi^{2}-\gamma}\right)(\sqrt{2} \sin (\ell \pi s))
$$


Applying Proposition 3.1 with $f(s)=\xi_{\ell}(s)$, we get

$$
\begin{aligned}
\Delta s \sum_{i=1}^{N-1} \xi_{\ell}\left(s_{i}\right)=\sum_{\mu=1}^{3} & \frac{(\Delta s)^{2 \mu} B_{2 \mu}}{(2 \mu) !}\left(\xi_{\ell}^{(2 \mu-1)}(1)-\xi_{\ell}^{(2 \mu-1)}(0)\right) \\
& -(\Delta s)^{6} \int_{0}^{1} \widetilde{B}_{6}(N s) \xi_{\ell}^{(6)}(s) \mathrm{d} s .
\end{aligned}
$$

From the homogeneous Dirichlet condition in (3.14), the compatibility condition (3.27) and (3.16) with $v=3$, we see that

$$
\xi_{\ell}^{\prime}(0)=\xi_{\ell}^{(3)}(0)=\xi_{\ell}^{\prime}(1)=\xi_{\ell}^{(3)}(1)=0 .
$$

Thus

$$
\begin{aligned}
& \left\|W\left(s_{i}\right)-W_{i}^{h}\right\|_{L_{h}^{2}(0,1)}^{2} \\
\leq & C N^{-12}\left(\sum_{\ell=1}^{N-1}\left(\frac{B_{6}}{6 !}\right)^{2}\left|\xi_{\ell}^{(5)}(1)-\xi_{\ell}^{(5)}(0)\right|^{2}+\sum_{\ell=1}^{N-1}\left|\int_{0}^{1} \widetilde{B}_{6}(N s) \xi_{\ell}^{(6)}(s) \mathrm{d} s\right|^{2}\right) .
\end{aligned}
$$

Moreover,

$$
\xi_{\ell}^{(5)}(0)=\frac{5 \sqrt{2} \ell \pi}{\ell^{2} \pi^{2}+\gamma} Z^{(4)}(0), \quad \xi_{\ell}^{(5)}(1)=\frac{(-1)^{\ell} 5 \sqrt{2} \ell \pi}{\ell^{2} \pi^{2}+\gamma} Z^{(4)}(1)
$$

Therefore

$$
\begin{aligned}
& \sum_{\ell=1}^{N-1}\left(\frac{B_{6}}{6 !}\right)^{2}\left|\xi_{\ell}^{(5)}(1)-\xi_{\ell}^{(5)}(0)\right|^{2} \\
\leq & C\|Z\|_{C^{4}[0,1]}^{2} \sum_{\ell=1}^{N-1}\left(\frac{\ell}{\ell^{2} \pi^{2}+\gamma}\right)^{2} \leq C\|Z\|_{C^{4}[0,1]}^{2} \leq C\|Z\|_{H^{6}(0,1)}^{2} .
\end{aligned}
$$

Using the same argument as in the proof of (5.2a), one can estimate the second term on the right hand side of (5.22) to get

$$
\begin{aligned}
& \sum_{\ell=1}^{N-1}\left|\int_{0}^{1} \widetilde{B}_{6}(N s) \xi_{\ell}^{(6)}(s) \mathrm{d} s\right|^{2} \\
\leq & C\left(\|W\|_{H^{6}(0,1)}^{2}+\|Z\|_{C^{4}[0,1]}^{2}+\|Z\|_{H^{6}(0,1)}^{2}\right) \leq C\|Z\|_{H^{6}(0,1)}^{2} .
\end{aligned}
$$

Thus the proof for $(5.2 b)$ is complete. So, the theorem is proved.

\section{Acknowledgments}

The research of Liu was supported by the NSF grant DMS 10-11738. The research of Wang was supported by National Science Council of Taiwan under grant 97-2115-M-007005. In addition, this work is also supported in part by National Center for Theoretical Sciences of Taiwan. 


\section{References}

[1] F. Auteri and L. Quartapelle, Spectral solvers for spherical elliptic problems, J. Comput. Phys., 227 (2007), 36-54.

[2] M. H. Carpenter, D. Gottlieb and S. Abarbanel, Stable and accurate boundary treatments for compact, high-order finite difference schemes, Appl. Numer. Math., 12(1-3) (1993), 55-87.

[3] W. E and J.-G. Liu, Essentially compact schemes for unsteady viscous incompressible flows, J. Comput. Phys., 126 (1996), 122-138.

[4] B. Fornberg, A Practical Guide to Pseudo-Spectral Methods, Cambridge University Press, New York, 1998.

[5] G. A. Glatzmaier and P. H. Roberts, A three-dimensional convective dynamo solution with rotating and finitely conducting inner core and mantle, Int. Phys. Earth. Planet., 91 (1995), 63-75.

[6] D. Gottlieb and S. A. Orszag, Numerical Analysis of Spectral Methods Theory and Applications, SIAM, Philadelphia, 1977.

[7] J. S. Hesthaven, S. Gottlieb and D. Gottlieb, Spectral Methods for Time-Dependent Problems, Cambridge University Press, New York, 2007.

[8] M. Li, T. Tang and B. Fornberg, A compact fourth-order finite difference scheme for the steady incompressible Navier-Stokes equations, Int. J. Numer. Method. Fluids., 20 (1995), 1137-1151.

[9] S. Orszag, Fourier series on spheres, Weather. Rev., 102 (1974), 56-75.

[10] R. Kress, Numerical Analysis, Springer, New York, 1998.

[11] C. Ronchi, R. Iacono and P. S. Paolucci, The "cubed sphere": A new method for the solution of partial differential equations in spherical geometry, J. Comput. Phys., 124 (1996), 93-114.

[12] J. Shen, Efficient spectral-Galerkin methods IV, spherical geometries, SIAM J. Sci. Comput., 20 (1999), 1438-1455.

[13] J. Shen and T. Tang, Spectral and High-Order Methods with Applications, Science Press, Beijing, 2006.

[14] A. Tilgner, Spectral methods for the simulation of incompressible flows in spherical shells, Int. J. Numer. Method. Fluids., 30 (1999), 713-724.

[15] T. Wiegelmann, Computing nonlinear force-free coronal magnetic fields in spherical geometry, Solar. Phys., 240 (2007), 227-239.

[16] S. Y. K. Yee, Solution of Poisson's equation on a sphere by truncated double Fourier series, Month. Weather. Rev., 109 (1981), 501-505.

[17] M. Yoshida and A. Kageyama, Application of the Yin-Yang grid to a thermal convection of a Boussinesq fluid with infinite Prandtl number in a three-dimensional spherical shell, Geophys. Res. Lett., 31 (2004), L12609. 